\title{
Interference Analysis for an Aeronautical Mobile Airport Communications System
}

\author{
Jeffrey D. Wilson \\ NASA Glenn Research Center \\ Cleveland, Ohio, USA \\ $+12164333513$ \\ Jeffrey.D.Wilson@nasa.gov \\ Robert J. Kerczewski \\ NASA Glenn Research Center \\ Cleveland, Ohio, USA \\ $+12164333434$ \\ rkerczewski@nasa.gov
}

\begin{abstract}
The next generation of aeronautical communications for airport surface applications has been identified through a NASA research program and an international collaborative future communications study. The result, endorsed by both the United States and European regulatory agencies is called AeroMACS (Aeronautical Mobile Airport Communications System) and is based upon the IEEE 802.16e mobile wireless standard. Coordinated efforts to develop appropriate aviation standards for the AeroMACS system are now underway within RTCA (United States) and Eurocae (Europe). AeroMACS will be implemented in a recently allocated frequency band, 5091$5150 \mathrm{MHz}$. As this band is also occupied by fixed satellite service uplinks, AeroMACS must be designed to avoid interference with this incumbent service. The aspects of AeroMACS operation that present potential interference to the fixed satellite service are under analysis in order to enable the definition of standards that assure that such interference will be avoided. The NASA Glenn Research Center has been involved in this analysis, and the first results of modeling and simulation efforts directed at this analysis are the subject of this paper. ${ }^{12}$
\end{abstract}

\section{TABLE OF CONTENTS}

1. INTRODUCTION. . .1

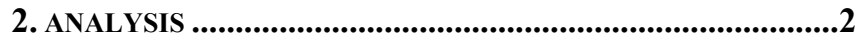

3. RESULTS ........................................................................................

4. CONCLUSION ................................................................................

5. ACKNOWLEDGMENTS ........................................................

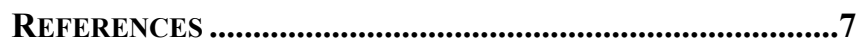

BIOGRAPHY ....................................................................................

\section{INTRODUCTION}

Next generation air transportation systems will achieve high levels of efficiency and safety through a combination of new air traffic management methods, automation, and collaboration, requiring an underlying information infrastructure providing major increases in communications

\footnotetext{
${ }^{1}$ U. S. Government work not protected by U. S. copyright.

${ }^{2}$ IEEEAC paper \#1098, Version 1, December 10, 2010.
}

capacity and performance. The Eurocontrol-FAA Future Communications Study has confirmed that a single communications system connecting the aircraft to the air traffic management system in all domains of flight (airport, terminal area, en-route, and oceanic/remote/polar) is not feasible. Due to physical and operational differences in the different flight domains a combination of several types of communications links is required. For the airport surface, the study recommended a wireless communications network based on the IEEE 802.16e mobile wireless standard [1].

In order to enable a safe and reliable airport surface communications network that is interoperable between airports both nationally and globally, a development and test program as well as the development of technical standards are being undertaken. This includes a test facility now in operation at the Cleveland Hopkins International Airport and the adjacent NASA Glenn Research Center (GRC) known as the Cleveland CNS Testbed. The testbed includes a prototype airport surface wireless communications network based on the IEEE 802.16e standard, known as Aeronautical Mobile Airport Communications System (AeroMACS). The testbed enables system tests and demonstrations providing technical support for the definition of the AeroMACS standard [2].

The AeroMACS system is envisioned as a multi-node wireless network covering all areas of the airport surface and, if necessary, areas beyond the airport proper where facilities and equipment such as lighting, navigational aids, weather sensors and wake sensors might need to be located. The system would accommodate all mobile communications requirements including aircraft, various types of ground vehicles, and personnel, and also enable links to fixed airport assets. Aircraft that are parked at a gate or taxiing would also be able to access the system, as long as the aircraft is not in flight.

The planned airport surface network will be scalable for small, medium and large airports through the use of one or more network nodes, or cells. It will also be interconnected with existing wired network infrastructure to create a hybrid 
network communications capability with enhanced reliability. For airports without existing wired network infrastructure, the wireless communications network system infrastructure can be installed much more easily and at far lower cost than wired infrastructure when such communications network capability is required.

AeroMACS is based upon the IEEE 802.16e standard, which provides several needed capabilities. It can maintain connection with moving vehicles at speeds up to $120 \mathrm{~km} / \mathrm{hr}$ so that taxiing aircraft as well as other airport surface vehicles can enter the network. It can work with mobile users in non-line-of-sight (NLOS) conditions, an essential element for an airport surface environment with many large moving aircraft. It can provide up to $50 \mathrm{Mbps}$ in the 2-6 $\mathrm{GHz}$ band, which matches the available spectrum as described below, in cells of radius 1-3 miles appropriate for a mesh network architecture on an airport surface.

The 802.16e standard also has a number of other desirable features including: Orthogonal Frequency Division Multiple Access (OFDMA) with variable power, spreading and reuse; support of a full range of smart antenna technologies such as beam forming, space-time code, and spatial multiplexing; support of hard-handoff, fast base station switching and macro diversity handover; and support of multicast and broadcast service. Adaptive modulation and coding, using QPSK, 16QAM and 64QAM, and convolutional turbo code with variable code rate are among the $802.16 \mathrm{e}$ options which enable adaptation to link conditions to maintain a high quality of service (QoS). Multiple device/user authentication, flexible key management protocol, strong traffic encryption, control and management plane message protection and security protocol optimizations for fast handovers are available to provide the necessary data and network security.

The development of national and international standards for AeroMACS is underway with activities in the United States (RTCA Special Committee 223) and Europe (Eurocae Working Group 82). AeroMACS is intended to be a globally interoperable standard to provide several categories of aeronautical communications capabilities for airport surface aviation safety applications. It is intended to operate in the 5091-5150 MHz band, for which a new Aeronautical Mobile (Route) Service (AM(R)S) allocation was approved at the 2007 World Radiocommunications Conference. The $\mathrm{AM}(\mathrm{R}) \mathrm{S}$ service allocation in this band is specifically limited to airport surface applications.

However, this new $\mathrm{AM}(\mathrm{R}) \mathrm{S}$ band also has several other approved services. New services must coordinate between themselves to avoid mutual interference. But of greater concern is an existing user in this band, operating in the fixed satellite service (FSS). In particular, mobile satellite service feeder links are now operating within this band. An example of such a user is the Globalstar system. The AeroMACS service must be designed and standardized so as to assure no interference with this existing service.
Interference issues related to the operation of an aeronautical mobile airport communications system (AeroMACS) in the C-Band (specifically 5091-5150 MHz) in general are being investigated as part of the standards development process. The issue of primary interest is cochannel interference from AeroMACS into mobile-satellite system (MSS) feeder uplinks. The investigation is focusing on establishing practical limits on AeroMACS transmissions from airports so that the threshold of interference into MSS feeder links is not exceeded.

NASA Glenn Research Center is contributing to this investigation through the development of software models of AeroMACS installations at airports distributed throughout the United States and adjacent areas. Simulation models using the software package Visualyse Professional, developed by Transfinite Systems Limited, have been built to analyze this interference scenario.

An initial set of results using basic AeroMACS parameters have been completed. The models used and results obtained are presented, with comparisons to interference results developed by MITRE-CAASD. These models have been shown to provide realistic analyses of emerging AeroMACS designs to be developed from NASA Cleveland Test Bed, RTCA SC-223, and European results, and enable the development of standards that ensure that AeroMACS can operate without presenting unacceptable interference to other systems in the 5091-5150 MHz band.

\section{ANALYSIS}

In this interference modeling of several simplified AeroMACS architectures, Visualyse Professional Version 7 software from Transfinite Systems Limited (UK) [3] is utilized. This software is a powerful and flexible tool that can be used to simulate a wide range of radiocommunication systems and the interference between them. The components of the models used in this study consisted of antennas, stations, carriers, links, and interference paths and were created with the following nine steps.

\section{Define antennas}

The three-dimensional gain directivities of the antennas are defined either through predefined masks or through userentered tables. In these models, the gain of both the omnidirectional and sectoral antennas are entered with tables.

\section{Locate stations}

The stations are the stationary or dynamic geographic locations of the transmitters and receivers. These models have stationary transmitter stations at each of the airports and a single stationary reference receiver station at the Globalstar low earth orbit altitude of $1414 \mathrm{~km}$. This reference receiver station is located at arbitrary longitude and latitude values which are later moved over the geographic range of interest to determine the power flux density at low earth orbit. 


\section{Specify carriers}

Carriers define the bandwidth and polarizations of the signal spectrum. In these models, the polarization is linear horizontal and the occupied bandwidth is $1.23 \mathrm{MHz}$ for the Globalstar carrier and 5, 10, or $20 \mathrm{MHz}$ for the airport transmitter carriers.

\section{Set up links}

Links define the communications paths between stations. For each link, the associated transmitter antenna, receiver antenna, carrier, center frequency, and transmit power is assigned. These models include transmitter links for each of the airports with the airport transmitter carrier at a center frequency of $5.1 \mathrm{GHz}$. The receiver link at the Globalstar satellite uses the Globalstar carrier also at the center frequency of $5.1 \mathrm{GHz}$.

\section{Set up propagation environment}

Visualyse enables the user to choose from a large number of ITU-R recommended propagations models, which can include effects such as diffraction over terrain and rain loss. For these models, ITU-R Rec. P. 525, Basic Transmission Loss in Free Space, is used.

\section{Set up interference paths}

An interference path assignment enables the calculation of the interference from the interfering links into the victim link. In these models, the victim link is the receiver link at the Globalstar satellite and the interfering links are the transmitter links from each of the airports.

\section{Specify output desired}

Visualyse can calculate statistics for any of the interference parameters $\mathrm{C}, \mathrm{N}, \mathrm{I}, \mathrm{C} / \mathrm{I}, \mathrm{C} / \mathrm{N}, \mathrm{C} /(\mathrm{N}+\mathrm{I}), \mathrm{I} / \mathrm{N}, \mathrm{PFD}$, and EPFD. Threshold values can be defined for these parameters to determine the percent time interference is observed. In these models, the parameter I, interference power, is calculated with a threshold value of $-157.3 \mathrm{dBW}$.

\section{Run}

A typical run for these models requires about 40 minutes on a PC with a $3.0 \mathrm{GHz}$ CPU.

\section{Analyze results}

Visualyse enables parameter values at a specific time or statistical values over a time range to be examined. A wide range of graphs can be generated including data vs. time, scatter plots, and distribution charts. In these models, the Area Analysis feature was used to produce the color contours of the interference power over the North America area shown in the figures below.

Further details of these steps are described in [4].

\section{RESULTS}

\section{Omni-directional Antennas}

In order to benchmark the software, the architecture configuration of a case from Hoh, Gheorghisor and Box [5] is used in the model and the results compared. In [5], the co-channel interference from an ANLE (Airport Network and Location Equipment / now referred to as AeroMACS) system to non-geostationary mobile-satellite-service (MSS) feeder uplinks (Globalstar) was analyzed. It was assumed that 5.8 watts was transmitted with an omni-directional antenna at each of 497 major airports in the contiguous United States. A worst case scenario was modeled with all transmitters on $100 \%$ of the time. A $20 \mathrm{MHz}$ bandwidth channel was assumed for the airport transmitter carriers. The aggregate interference power at Low Earth Orbit (1414 $\mathrm{km}$ above the surface) was calculated and a global view of the results is shown in Figure 1.



Figure 1. Global view of simulated aggregate interference power at LEO from 497 airports each with an omnidirectional antenna transmitting 5.8 watts. The scale is dBW.

The corresponding two-dimensional view is shown in Figure 2 and the corresponding view from [5] is shown in Figure 3.

The agreement is excellent between the Visualyse simulated interference power distribution shown in Fig. 2 and that calculated by Hoh, et al. in [5] and shown in Fig. 3 . (Different color schemes are used, with the yellow-green boundary representing the threshold boundary of -155.5 dBW in Fig. 2 corresponding to the red-green boundary in Fig 3.) In the Visualyse model, the maximum interference 


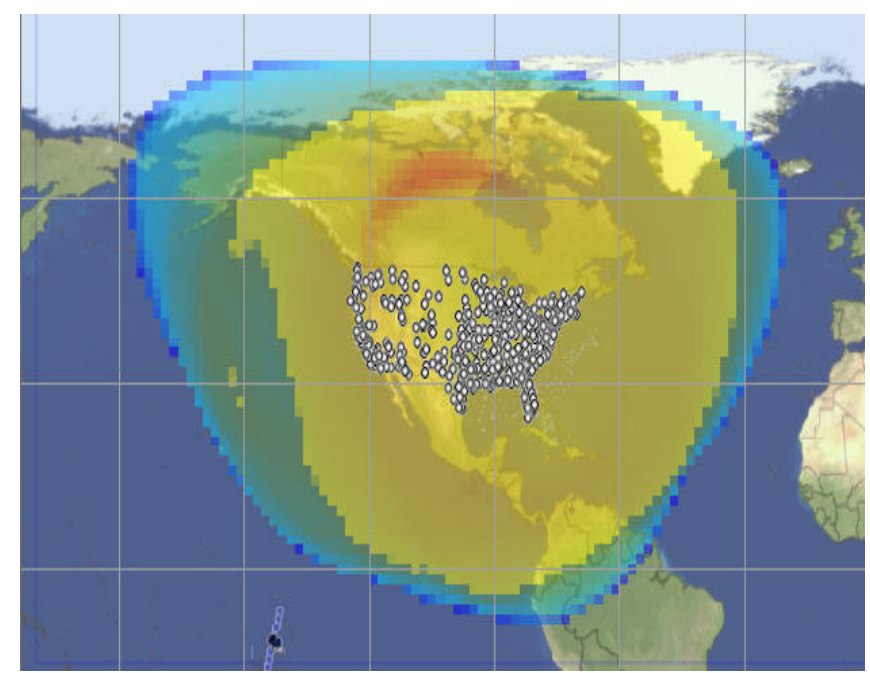

Figure 2. Simulated interference power in two-dimensional view.

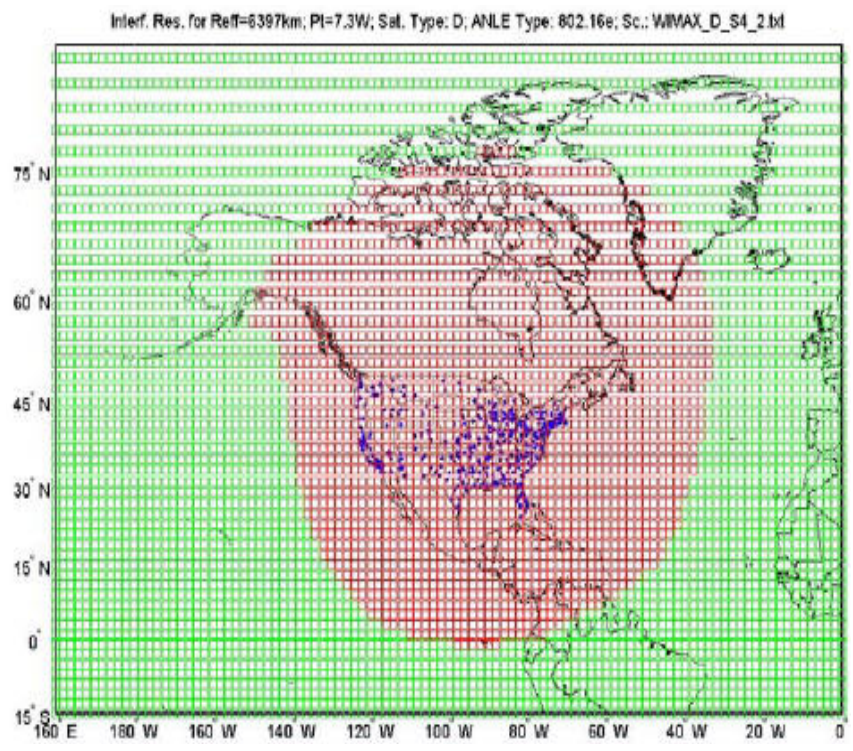

Figure 3. Simulated interference power from Hoh, et al. [4].

power is $-149.3 \mathrm{dBW}$ at the location $64^{\circ} \mathrm{N} 105^{\circ} \mathrm{W}$, which compares well with the value of $-150.0 \mathrm{dBW}$ in [5] at the location $67^{0} \mathrm{~N} 104^{0} \mathrm{~W}$. (Note that the threshold interference power value has since been tightened to $-157.3 \mathrm{dBW}$ corresponding to a $2 \%$ increase of the satellite receiver's noise temperature [6].)

In order to keep the interference power under the new threshold value of $-157.3 \mathrm{dBW}$ everywhere, the transmitted power at each airport needs to be decreased to $891 \mathrm{~mW}$ with the resulting interference power distribution shown in Figure 4.

The channel bandwidths now being considered for AeroMACS are $10 \mathrm{MHz}$ and $5 \mathrm{MHz}$. The threshold

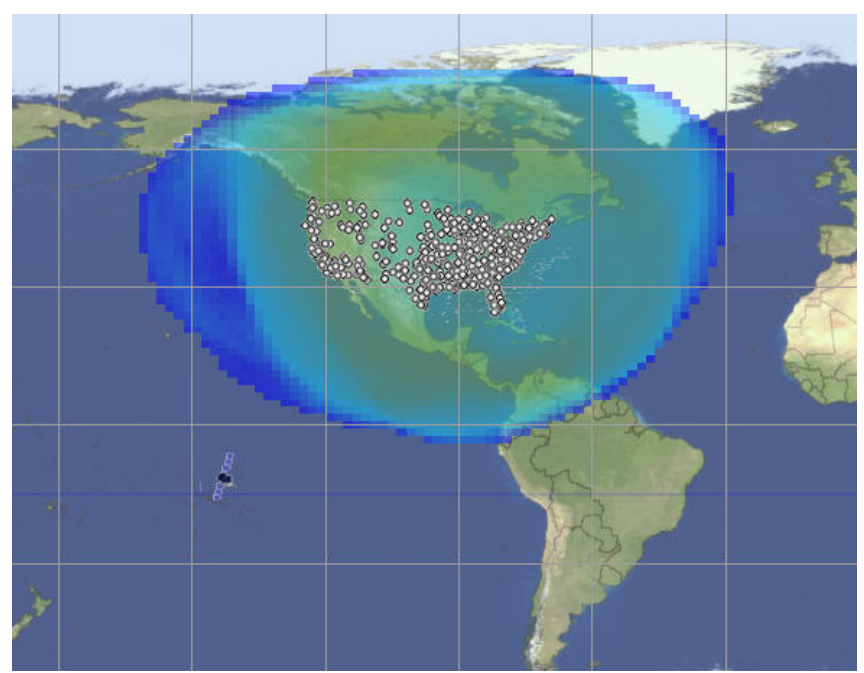

Figure 4. Simulated interference power is decreased below threshold everywhere when transmitted power decreased to $891 \mathrm{~mW}$ at each airport with $20 \mathrm{MHz}$ channel bandwidth.

transmitted power is linearly proportional to channel bandwidth, so at 10 and $5 \mathrm{MHz}$, the threshold transmitted powers are $447 \mathrm{~mW}$ and $224 \mathrm{~mW}$, respectively.

In the model, it was assumed that all the airports were at sea level. In order to determine if airport altitude needs to be included in the model, the interference power from just the Denver airport was simulated with the altitude at sea level and at $1.6 \mathrm{~km}$. The results for the two cases are shown to be virtually identical in Figures 5 (a) and (b). Thus airport altitude does not have a significant impact on interference power at low earth orbit and can be ignored.

The effect of adding additional airports was investigated by increasing the number by $52.3 \%$ from 497 to 757 . The new set includes all of the 703 FAA towered airports and heliports not only in the contiguous United States but also those in Alaska, Hawaii, and the Caribbean. Additionally 34 Canadian and 20 Mexican airports are included. The resulting interference power distributions for the two cases are shown in Figure 6 for a $20 \mathrm{MHz}$ bandwidth channel. The effect of adding significant additional airports is to increase the interference power density but not as much as might have been expected. The maximum transmitted power at each airport to remain under the $-157.3 \mathrm{dBW}$ threshold decreases only by $10.3 \%$ from the model with 497 airports to the one with 757 airports. Thus for 5, 10, and $20 \mathrm{MHz}$ channels, the maximum power with 757 airports is 799, 401, and $201 \mathrm{~mW}$ respectively.

\section{Sectoral Antennas}

Sectoral antennas can provide more targeted coverage than can omni-directional antennas. Examples of this are shown in Figure 7 for (a) single beam and (b) two beam configurations. Configurations with one, two and three 


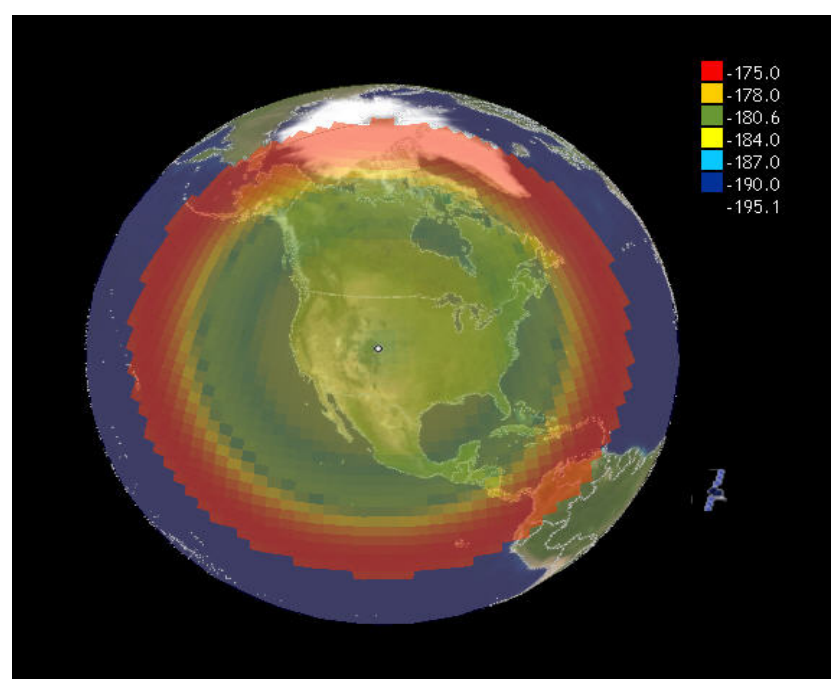

(a)

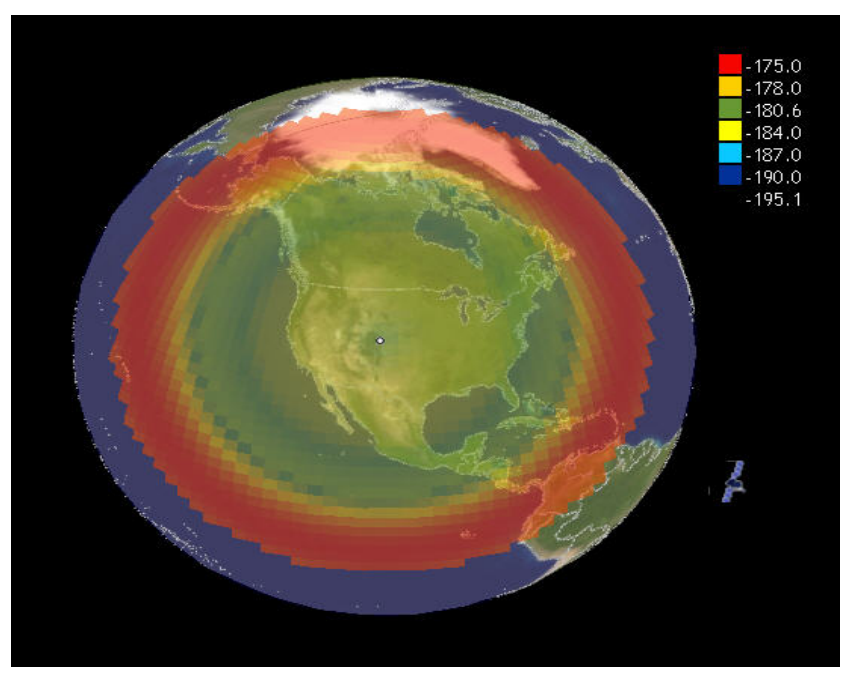

(b)

Figure 5. Simulated interference power distribution from an omni-directional antenna at Denver airport modeled at (a) sea level and (b) $1.6 \mathrm{~km}$ with an omni-directional antenna transmitting 5.8 watts and a $20 \mathrm{MHz}$ bandwidth channel.

beams/transmitters were considered. The beams were randomly directed at each of the 757 airports, with the beams separated by $120^{\circ}$ in the two and three beam configurations.

For each configuration, both $90^{\circ}$ and $120^{\circ}$ beam widths were considered. Figure 8 shows the antenna gain as a function of (a) azimuth and (b) elevation angle [7] for a maximum of $15 \mathrm{dBi}$. It is seen that the $120^{\circ}$ beamwidth case has a gain that is broader in the azimuth direction, but narrower in elevation. Thus it produces less radiation at low earth orbit and the maximum transmitted power to remain under

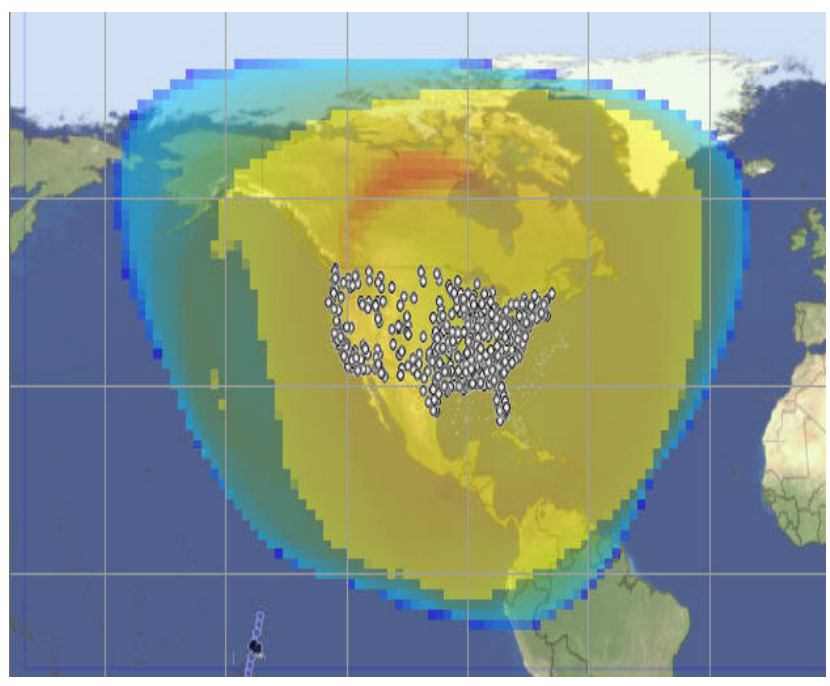

(a)

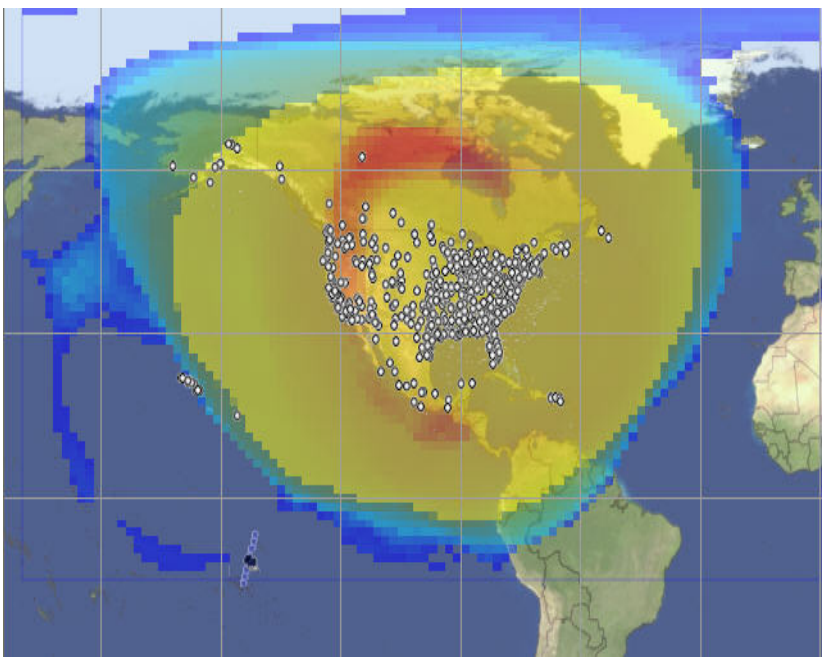

(b)

Figure 6. Simulated interference power distribution from (a) 497 airports and (b) 757 airports with omni-directional antennas transmitting 5.8 watts and a $20 \mathrm{MHz}$ channel.

threshold is higher in all configurations. From the values in Table 1, the maximum transmitted power is about $17 \%$ higher for the $120^{\circ}$ beamwidth case. Also from Table 1, the maximum allowable power transmission per airport is approximately independent of the number of beams.

Table 1. Maximum Transmitted Power per Beam to Remain Under Threshold Everywhere with $5 \mathrm{MHz}$ Channels

\begin{tabular}{|l|c|c|c|}
\hline & 1 Beam & 2 Beams & 3 Beams \\
\hline $90^{0}$ Beamwidth & $141.8 \mathrm{~mW}$ & $70.4 \mathrm{~mW}$ & $47.4 \mathrm{~mW}$ \\
\hline $120^{\circ}$ Beamwidth & $166.0 \mathrm{~mW}$ & $83.2 \mathrm{~mW}$ & $55.3 \mathrm{~mW}$ \\
\hline
\end{tabular}




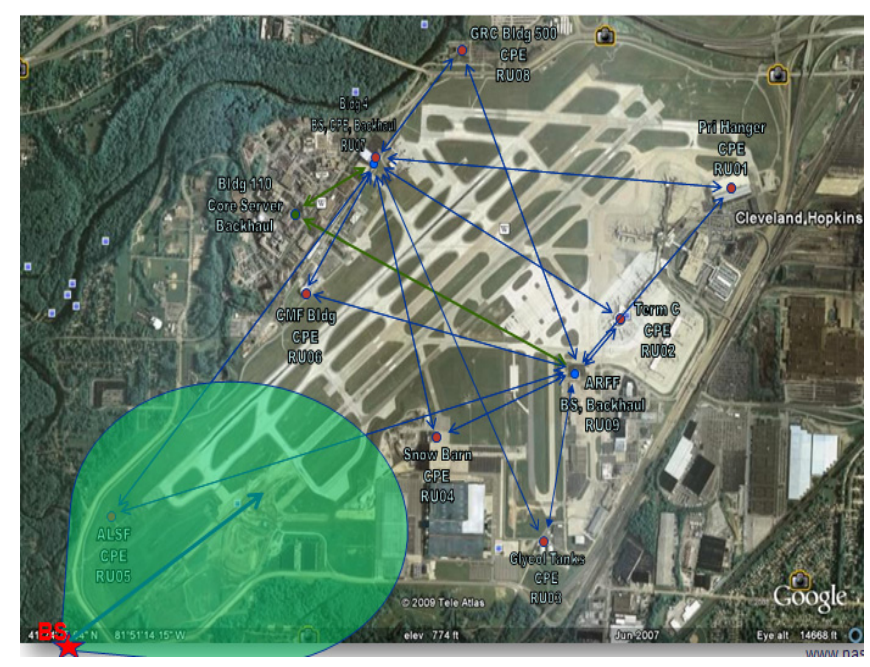

(a)

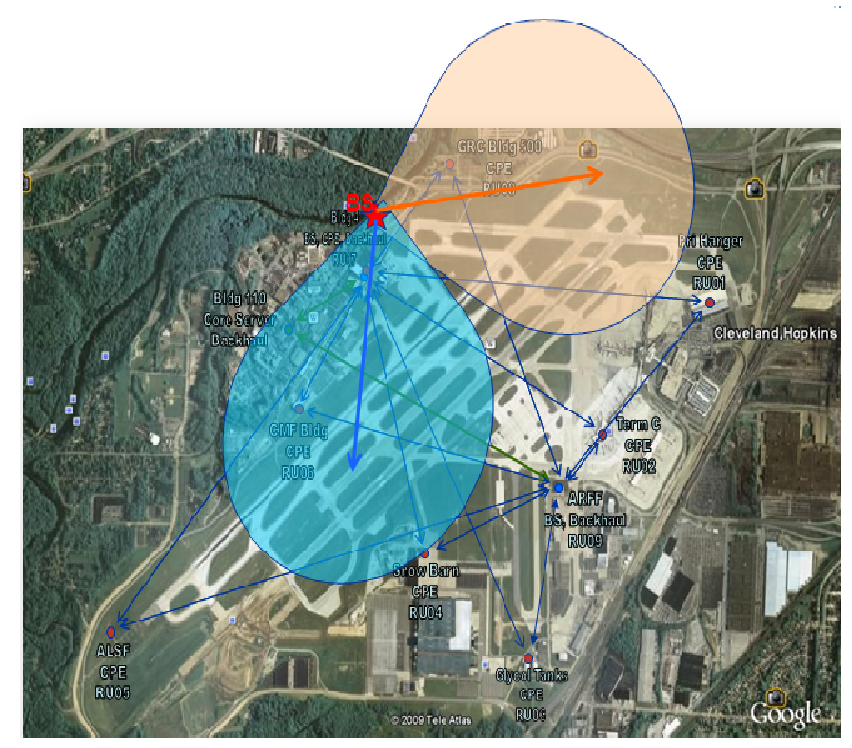

(b)

Figure 7. Examples of (a) single beam and (b) two beam sectoral antenna configurations.

Table 2. Maximum Transmitted Power per Beam to Remain Under Threshold Everywhere with $5 \mathrm{MHz}$ Channels for 3Beam Configuration, as a Function of Antenna Tilt.

\begin{tabular}{|l|l|l|l|l|l|}
\hline Tilt & $0^{0}$ & $-1^{0}$ & $-2^{0}$ & $-3^{0}$ & $-4^{0}$ \\
\hline $\begin{array}{l}90^{0} \\
\text { beamwidth }\end{array}$ & $\begin{array}{l}47.4 \\
\mathrm{~mW}\end{array}$ & $\begin{array}{l}48.9 \\
\mathrm{~mW}\end{array}$ & $\begin{array}{l}50.0 \\
\mathrm{~mW}\end{array}$ & $\begin{array}{l}50.5 \\
\mathrm{~mW}\end{array}$ & $\begin{array}{l}51.0 \\
\mathrm{~mW}\end{array}$ \\
\hline $\begin{array}{l}120^{0} \\
\text { beamwidth }\end{array}$ & $\begin{array}{l}55.3 \\
\mathrm{~mW}\end{array}$ & $\begin{array}{l}60.4 \\
\mathrm{~mW}\end{array}$ & $\begin{array}{l}63.9 \\
\mathrm{~mW}\end{array}$ & $\begin{array}{l}67.0 \\
\mathrm{~mW}\end{array}$ & $\begin{array}{l}68.1 \\
\mathrm{~mW}\end{array}$ \\
\hline
\end{tabular}

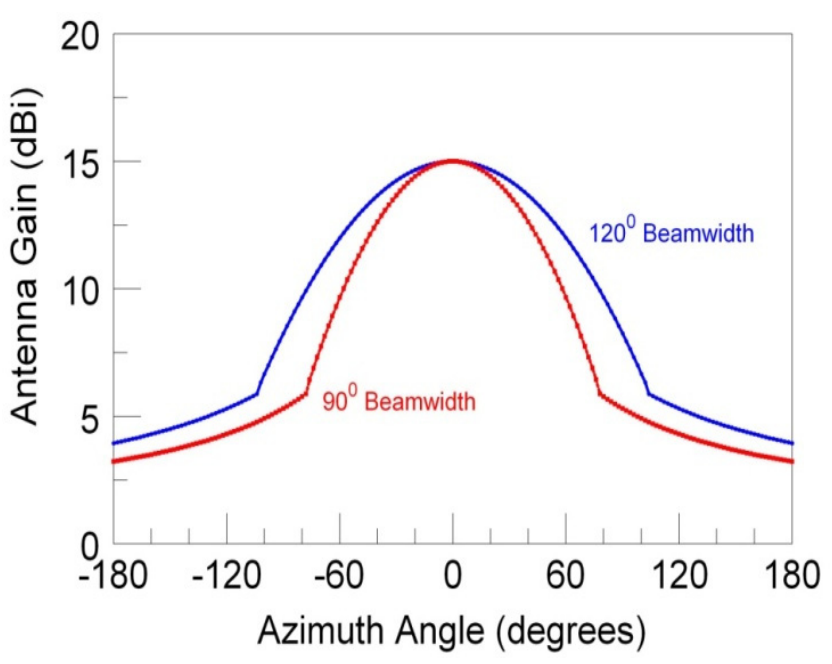

(a)

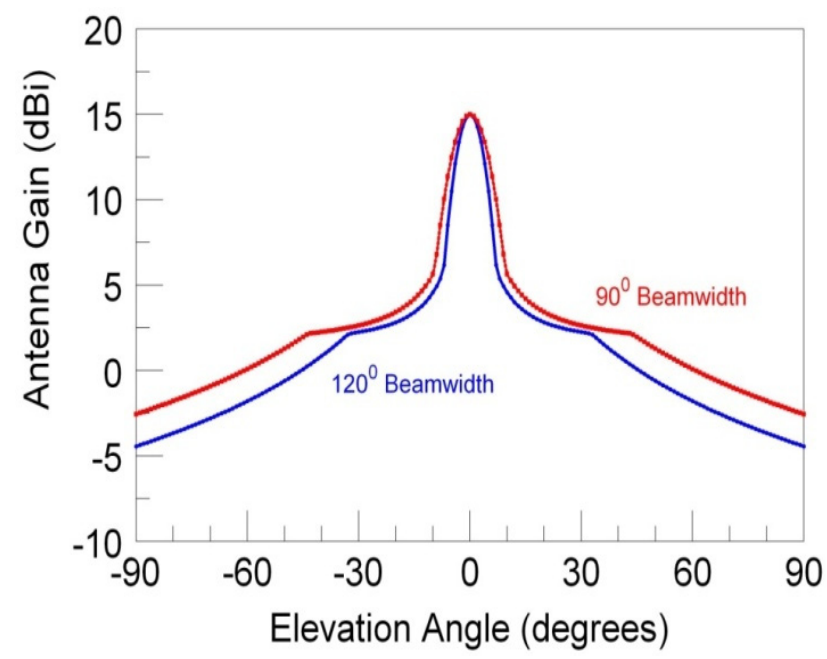

(b)

Figure 8. Antenna gain dependence on (a) azimuth angle and (b) elevation angle for $90^{\circ}$ and $120^{\circ}$ beamwidth sectoral antennas[7].

A method to reduce the upward propagation and interference from the AeroMACS antennas is to introduce a downward tilt to the antennas. This effect was modeled with the 3-beam configuration and the results are shown in Table 2. It is seen that the benefit from downward tilt is almost three times stronger for the $120^{\circ}$ beamwidth antenna. For example with a $-2^{0}$ tilt, the maximum allowable transmitted power increases by only $5.5 \%$ for the $90^{\circ}$ beamwidth, but by $15.5 \%$ for the $120^{\circ}$ beamwidth. 


\section{Conclusion}

NASA Glenn Research Center is participating in investigations of the performance of the AeroMACS system design in order to enable the development of standards for the future deployment of the system. In this paper we have described the analysis of the interference of AeroMACS into mobile satellite service (MSS) uplinks that are allocated as co-primary users of the intended AeroMACS frequency band of 5091-5150 MHz.

In order to help establish practical limits on AeroMACS transmissions from airports such that the threshold of interference into the MSS uplink is not exceeded, suitable for inclusion in RTCA and Eurocae standards now under development, interference models have been created with Visualyse Professional software. A baseline model scenario consisted of a single omni-directional transmitting antenna at each of the 497 major contiguous United States airports. The results agreed closely with those of a previous study in which the interference power over almost all of North America exceeds the desired threshold. In order to keep the interference power under the new threshold value of -157.3 $\mathrm{dBW}$ everywhere, the transmitted power at each airport needs to be decreased to $891 \mathrm{~mW}$ for $20 \mathrm{MHz}$ channels. The threshold value is linearly proportional to channel bandwidth, so at 10 and $5 \mathrm{MHz}$, the threshold transmitted powers are $447 \mathrm{~mW}$ and $224 \mathrm{~mW}$, respectively.

The effect of adding additional airports was investigated by increasing the number from 497 to 757 . The additional airports were not only from the contiguous United States, but also from Alaska, Hawaii, the Caribbean, Canada, and Mexico. The effect of adding significant additional airports is to increase the interference power density but not as much as might have been expected. Increasing the number of airports by $50 \%$ decreased the maximum transmitted power at each airport to remain under threshold by only about $10 \%$.

Sectoral antennas with both $90^{\circ}$ and $120^{\circ}$ beamwidths were considered. From strictly an interference point of view, the $120^{\circ}$ beamwidth sectoral antennas are superior and allow the maximum transmitted power to be about $17 \%$ higher. This advantage is enhanced further if downward antenna tilt is introduced to reduce the upward component of propagation. While a downward $-2^{0}$ tilt allows the maximum transmitted power to increase by only $5.5 \%$ for the $90^{\circ}$ beamwidth sectoral antenna it allows an increase of $15.5 \%$ for the $120^{\circ}$ beamwidth sectoral antenna.

These analyses have yielded results indicating that current draft AeroMACS standards can provide effective operation of the system without interfering with the co-channel MSS uplinks. Nevertheless, the maximum power transmission levels indicated by these results provide a significant constraint on the design of the system and the underlying standards, such that parameters including number of sites, cells per site, antenna design and pointing angle need to be carefully specified within the standards. Further analysis work will continue, including such activities as development of airport AeroMACS deployment models and detailed data traffic load models.

\section{ACKNOWLEDGMENTS}

The authors wish to thank John Pahl of Transfinite Systems Limited for help with the Visualyse Professional software, Dr. Izabela Gheorghisor of The MITRE Corporation for sharing her airport data set and helpful discussions, and James Budinger of NASA and Rafael Apaza of the Federal Aviation Administration for helpful discussions and comments.

\section{REFERENCES}

[1] R. J. Kerczewski, J. M. Budinger, and T. J. Gilbert, "Technology Assessment Results of the Eurocontrol/FAA Future Communications Study", 2008 IEEE Aerospace Conference, March, 2008.

[2] W. Ward, J. Budinger, R. Dimond, J. Wilson, and R. Apaza, "Aeronautical Mobile Airport Communications System Development Status" , IEEE 2010 Integrated Communications, Navigation and Surveillance Conference, May 11-13, 2010.

[3] http://www.transfinite.com/.

[4] J. D. Wilson, "Modeling C-Band Co-Channel Interference from AeroMACS Omni-Directional Antennas to Mobile Satellite Service Feeder Uplinks," NASA-TP (in publication).

[5] Y.-S. Hoh, I. L. Gheorghisor, and F. Box, "Feasibility Analysis of 5091-5150 MHz Band Sharing by ANLE and MSS Feeder Links," MITRE-CAASD Report MP 05W0000083, 2005.

[6] I. L. Gheorghisor, Y.-S. Hoh, and A. E. Leu, "Analysis of ANLE Compatibility with MSS Feeder Links," MITRECAASD Report MTR090458, 2009.

[7] ITU-R F.1336-2, "Reference radiation patterns of omnidirectional, sectoral and other antennas". 


\section{BIOGRAPHY}

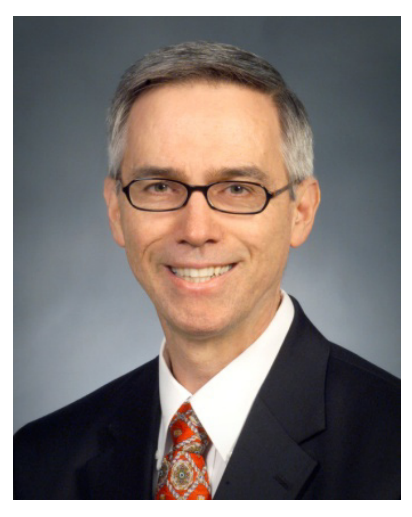

Jeffrey D. Wilson received the B.S. degree in physics magna cum laude from Bowling Green State University in 1976, and the M.S. and Ph.D. degrees in physics from the University of Illinois at Urbana-Champaign in 1978 and 1983, respectively. Since 1983, Dr. Wilson has been associated with the vacuum electronics microwave amplifier research group at NASA Glenn Research Center, Cleveland, Ohio. He spent the 1984-1985 academic year in postdoctoral study with the Air Force Thermionic Electronics Research (AFTER) Program at the University of Utah. His research efforts have focused on computational techniques to enhance the power, efficiency, and performance of coupledcavity, helical, and terahertz wave traveling-wave tubes (TWT'S), the electromagnetic properties of metamaterials, and interference issues in RF communications systems. Dr. Wilson is a Senior Member of IEEE.

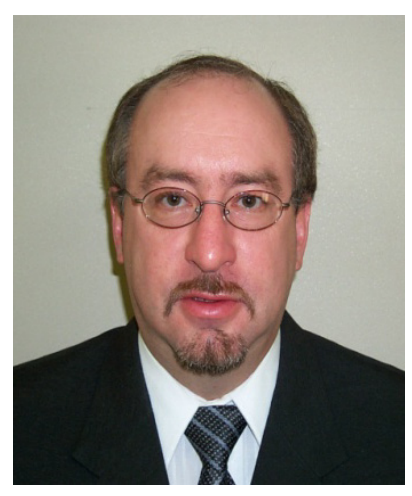

Robert J. Kerczewski has been involved with research and development of communications systems for space and aeronautical applications for the Analex Corporation (1982-1986) and NASA (1986-present). He holds a BEE degree from Cleveland State University (1982) and an MSEE degree from Case Western Reserve University (1987). He has served as Principle Investigator for ACTS Interference and Telemammography Experiments, and as Project Manager for the Advanced CNS Architectures and Systems Technologies, Space Based Technologies, and Integrated Vehicle Health Management Projects. He is currently engaged in aeronautical communications research activities for unmanned aerial vehicle command and control, airground communications, and vehicle health management. 